\title{
Evaluation of a Stepped Care Approach to Manage Depression and Diabetes Distress in Patients with Type 1 Diabetes and Type 2 Diabetes: Results of a Randomized Controlled Trial (ECCE HOMO Study)
}

\author{
Andreas Schmitt ${ }^{a, b}$ Bernhard Kulzer ${ }^{a, b, c} \quad$ André Reimer ${ }^{a} \quad$ Christian Herder $^{b}$, d, e \\ Michael Roden ${ }^{b}, d, e$ Thomas Haak ${ }^{a}$ Norbert Hermanns ${ }^{a, b}, c$

\begin{abstract}
aResearch Institute of the Diabetes Academy Mergentheim, Diabetes Center Mergentheim, Bad Mergentheim, Germany; ${ }^{\mathrm{b}}$ German Center for Diabetes Research (DZD), Muenchen-Neuherberg, Germany; ${ }^{\mathrm{C}}$ Department for Psychology, Otto-Friedrich-University of Bamberg, Bamberg, Germany; ${ }^{\mathrm{d} D e p a r t m e n t}$ of Endocrinology and Diabetology, Medical Faculty and University Hospital Duesseldorf, Heinrich-Heine-University Duesseldorf,

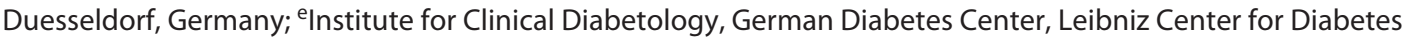
Research at Heinrich-Heine-University Duesseldorf, Duesseldorf, Germany
\end{abstract}

\section{Keywords}

Cognitive-behavioral therapy · Mood disorder - Depressive symptoms · Emotional distress · Stepped care approach

\begin{abstract}
Introduction: Depression is a common and serious complication of diabetes. Treatment approaches addressing the specific demands of affected patients are scarce. Objective: The aim of this work was to test whether a stepped care approach for patients with diabetes and depression and/or diabetes distress yields greater depression reduction than treatment-as-usual. Methods: Two-hundred and sixty patients with diabetes and elevated depressive symptoms $(C E S-D \geq 16)$ and/or elevated diabetes distress (PAID $\geq 40$ ) were randomized to stepped care for depression or diabetes treatment-as-usual. The primary outcome was the rate of meaningful depression reduction at the 12-month follow-up according to the HAMD (score $<9$ or reduction by $\geq 50 \%$ ). Secondary outcomes were changes in depression scores (HAMD/CES-D), diabetes distress (PAID), diabetes accep-
\end{abstract}

tance (AADQ), well-being (WHO-5), quality of life (EQ-5D/SF36), self-care behavior (SDSCA/DSMQ), $\mathrm{HbA}_{1 c}$, and biomarkers of inflammation. Results: One-hundred and thirty-one individuals were assigned to stepped care and 129 to treatment-as-usual. Overall, $15.4 \%$ were lost to follow-up. Meaningful depression reduction was observed in 80.2 versus $51.2 \%$ in stepped care versus treatment-as-usual ( $p<0.001$, intention-to-treat analysis). Of the secondary measures, the $\operatorname{HAMD}(\Delta-3.2, p<0.001)$, WHO-5 $(\Delta 1.5, p=0.007)$, and AADQ $(\Delta-1.0, p=0.008)$ displayed significant treatment effects, while effects on CES-D $(\Delta-2.3, p=0.065)$, PAID $(\Delta-3.5, p=$ $0.109)$, and SDSCA $(\Delta 0.20, p=0.081)$ were not significantly different. Both groups showed comparable changes in EQ$5 \mathrm{D} / \mathrm{SF}-36, \mathrm{DSMQ}, \mathrm{HbA}_{1 c}$, and biomarkers of inflammation (all $p \geq 0.19$ ). Conclusions: The stepped care approach improved depression, well-being, and acceptance. The results support that increasing treatment intensity on demand is effective and can help provide more optimal treatment. The inclusion of diabetes-specific interventions may be beneficial for patients with diabetes and elevated depression.

(c) 2021 The Author(s).

Published by S. Karger AG, Basel karger@karger.com www.karger.com/pps

Karger $\stackrel{\text { ' }}{5}$ BOPEN ACCESS
(C) 2021 The Author(s)

Published by S. Karger AG, Basel

This is an Open Access article licensed under the Creative Commons Attribution-NonCommercial-4.0 International License (CC BY-NC) (http://www.karger.com/Services/OpenAccessLicense), applicable to the online version of the article only. Usage and distribution for commercial purposes requires written permission.
Correspondence to:

Andreas Schmitt, schmitt@ diabetes-zentrum.de 


\section{Introduction}

The prevalence of depression in patients with diabetes is high. Up to $30 \%$ experience elevated depressive symptoms, while major depression is present in $11-13 \%$ according to meta-analytic evidence $[1,2]$. Additionally, one third of patients with diabetes experience high diabetes-related distress [3], which can occur independently of, concomitantly with, or in close relation with depressive symptomatology [4]; there is evidence suggesting a bidirectional etiological relationship between these conditions $[5,6]$.

Comorbid depression has been associated with an adverse impact on treatment and course of diabetes. Patients with diabetes and depression may experience problems of self-managing their diabetes optimally $[7,8]$, possibly resulting in a suboptimal glycemic outcome $[8,9]$. Longitudinal evidence supports that comorbid depression increases the risk of subsequent long-term complications of diabetes $[10,11]$; it is further associated with higher cardiovascular mortality [11]. In fact, mortality is already increased in patients with diabetes with minor depression [12]. Finally, the impact of combined diabetes and depression on quality of life is severe [13].

Suboptimal glycemia and increased risks of complications in patients with diabetes and depression may be explained by adverse self-management and health behaviors $[7,8]$. Another putative pathway is subclinical inflammation which is prevalent in this group $[14,15]$ and may affect glycemia, insulin sensitivity, and cardiovascular risks $[16,17]$. A prospective study of type 2 diabetes found subclinical inflammation mediating the relationship between depression and incident macrovascular complications [18]. Furthermore, subclinical inflammation might affect the depression treatment response in type 1 diabetes [19].

Diabetes distress, defined as the sum of emotional burdens, stresses, and worries due to the chronic condition, is one of the most frequent psychosocial concerns in patients with diabetes [20]. Diabetes distress increases the risk of developing depression $[5,6]$ and is associated with reduced quality of life $[20,21]$. Furthermore, it may affect self-management and glycemic outcome, similarly to depression [21]. A longitudinal study regarding type 2 diabetes found that depression and diabetes distress independently predicted less optimal self-management in different aspects [22]. There is also evidence suggesting that glycemic outcome may be worst in patients with diabetes experiencing depression and diabetes distress concomitantly [23].
Due to the complex interdependencies between diabetes and depression as well as the serious adverse impacts of diabetes-comorbid depression on quality of life outcomes, optimal diabetes treatment and glycemic outcome and finally the long-term "hard endpoints" of the disease, effective interventions for treating depression specifically in patients with diabetes are urgently needed. However, despite this requirement, only few diabetesspecific interventions have been developed [24]. Furthermore, due to the negative impact of comorbid depression and diabetes distress on health outcomes, international guidelines recommend regular screening for reduced well-being and depressive symptoms as well as specialized treatment for those identified with mental disorders such as major depression $[25,26]$. However, there is no consensus as to how the groups of patients with subclinical depression and/or high diabetes distress should be optimally treated: on one hand, it is evident that these aspects impact on quality of life and may affect diabetes outcomes, on the other hand, there is the risk of pathologizing people with non-clinical mental problems [27]. Also, standard depression treatment (i.e., psychotherapy/antidepressants) might constitute an over-treatment causing unnecessary side-effects and costs. Furthermore, it is unclear whether this approach is suitable for diabetes distress. Thus, a stepped care approach offering evidence-based treatments of increasing intensity sequentially might be optimal [27]: beginning with an intervention for depressive symptoms, the treatment is either ended or increased, depending upon remission or persistence.

Since the majority of patients with diabetes and mental health symptoms experience subclinical depression and/ or diabetes distress $[2,3]$, with these aspects bidirectionally interrelated [4] and diabetes distress predicting incident depression $[5,6]$, a diabetes-specific group treatment for depressive symptoms and diabetes distress constitutes a good starting point. Treating diabetes-specific distress in patients with diabetes and depression was found to improve depressive symptoms $[24,28]$. This approach may also suit patients with diabetes with mild-tomoderate depression, given functional impairment is non-severe and suicidal ideation is ruled out. Patients with diabetes not benefitting can then be offered more intensive treatment, with single cognitive-behavioral therapy (CBT) being first choice [27]. For patients with established and persistent mental conditions (such as major depression) in spite of previous, less intensive interventions, depression treatment consisting of intensified psychotherapy and/or antidepressant therapy might be 
required, as recommended by relevant guidelines for the treatment of clinical depressive disorders [25-27].

We developed a stepped care approach for patients with diabetes with depression and/or diabetes distress based on the above theoretical considerations. That is, patients receiving stepped care initially attended a five-session group treatment for depressive symptoms and diabetes distress, if there was no improvement they were then offered a single CBT intervention for major depression, and if still not improved were finally referred for standard outpatient depression treatment, as recommended [27]. A two parallel-group prospective randomized trial comparing the stepped care approach to a treatment-as-usual control condition was conducted to evaluate its efficacy in a 12-month follow-up (FU). As the primary outcome, the reduction of depression symptoms in the stepped care group compared to treatment-as-usual condition was analyzed. Furthermore, the effects of the stepped care approach on a broad range of secondary outcomes were analyzed. We expected beneficial effects of the treatment on diabetes distress, diabetes acceptance, emotional well-being, anxiety, quality of life, diabetes self-management, treatment satisfaction, and metabolic variables. Considering the possible role for subclinical inflammation in depression $[14,15]$, its impact on health outcomes [16-18] and potential moderation of depression treatment response [19], we assessed biomarkers of inflammation to evaluate possible changes following treatment and control for possible moderation of depression treatment.

\section{Materials and Methods}

\section{Study Ethics}

The study was approved by the Ethics Committee of the State Medical Chamber of Baden-Wuerttemberg (file No. F-2013-011) and carried out in accordance with the Declaration of Helsinki. All study participants provided written informed consent prior to inclusion. The study is registered at ClinicalTrials.gov (ID No. NCT01812291). The study is reported in accordance with the CONSORT guidelines for randomized controlled trials as well as methodological recommendations for trials of psychological interventions [29].

\section{Design}

This is a monocenter prospective randomized trial with two treatment arms. Participants were recruited at a specialized diabetes center (Diabetes Center Mergentheim, Germany) providing inpatient care to about 4,000 adults with diabetes annually; patients are referred there for comprehensive education and care or due to specific problems (e.g., persistent dysglycemia); the majority (approx. 55\%) has type 1 diabetes. Study participation included an initial inpatient phase of about 2 weeks followed by a 1 -year out- patient phase. The treatment-as-usual control group received diabetes care as usual at the diabetes center. The intervention group received diabetes care as usual plus the stepped care approach for depression. The first treatment step of the stepped care approach was provided during the inpatient phase, and further treatment steps during the outpatient phase. Treatment outcomes were evaluated pre- and post-treatment, after 6 months (interim), and in the decisive 12-month FU.

\section{Participant Enrolment}

All adults with type 1 diabetes or type 2 diabetes were approached by study staff on their second day at the center and invited to take part in a brief mental health screening with a questionnaire. The screening assessed depressive symptoms using the Center for Epidemiologic Studies Depression Scale (CES-D) and diabetes distress using the Problem Areas in Diabetes Scale (PAID) with commonly used cut-off scores of $\geq 16$ for the CES-D and $\geq 40$ for the PAID (i.e., identifying elevated depressive symptoms and elevated diabetes distress, respectively) $[3,5,18,20,21]$. The screening was implemented over a period of 18 months when the study sample was fully recruited. Participation in the screening was voluntary, and about $60 \%$ of invited patients returned their questionnaires. Positively screened patients were invited to an interview evaluating eligibility and assessing possible major depression using the MINI interview. Patients meeting the eligibility criteria were informed about the study and invited to participate. Patients identified with severe major depression (F32.2) were informed about this and offered counselling by a clinical psychologist but were excluded from study participation for ethical concerns. Eligible and consenting persons were randomly assigned to either stepped care or treatment-as-usual.

\section{Inclusion/Exclusion Criteria}

Eligible subjects were: patients with type 1 diabetes or type 2 diabetes; with elevated depressive symptoms $(C E S-D \geq 16)$ and/or elevated diabetes distress (PAID $\geq 40$ ); aged $18-70$ years. Patients were excluded if they had: current severe major depression (F32.2); an established diagnosis of psychotic, bipolar, personality, addictive, or severe eating disorder; current psychotherapy/antidepressant treatment; suicidal ideation; severe somatic illness (e.g., renal failure); inability to consent (based on records).

\section{Randomization}

Randomization was performed by the Coordination Centre for Clinical Trials (KKS) Duesseldorf. A pool of participants was established each week; if it reached 4-16 individuals by Friday, the KKS was contacted via fax and a person independent from the study randomized the participants to the groups with a 1:1 allocation ratio. Group allocation was non-blinded to participants and treating psychologists, while the outcome assessment was conducted by blinded study staff.

\section{Interventions}

The stepped care approach was developed by a group of clinical psychologists and healthcare professionals working in the field of diabetes (A.S., B.K., A.R., T.H., N.H., et al.). In an iterative development process, treatment steps were planned and corresponding interventions selected. For step 1, a diabetes-specific CBT-based group treatment for diabetes distress and depressive symptoms was selected. A previous trial had shown that the newly developed, 
Table 1. Steps and interventions of the stepped care approach

\begin{tabular}{|c|c|c|c|}
\hline Step No. & Treatment approach & Designated specific interventions & Received treatments and participation rates \\
\hline Step 1 & $\begin{array}{l}\text { Five sessions ( } 90 \text { min each) of } \\
\text { diabetes-specific CBT group } \\
\text { treatment focusing on diabetes- } \\
\text { related problems and distress and } \\
\text { depressive symptoms. Groups } \\
\text { comprised } 2-5 \text { subjects }\end{array}$ & $\begin{array}{l}\text { - Diabetes-specific problem analysis } \\
\text { - Explanatory model of diabetes distress and } \\
\text { depressive symptoms (bidirectional relationships } \\
\text { and impact on outcomes) } \\
\text { - Diabetes-related problem solving training } \\
\text { - Cognitive restructuring of dysfunctional } \\
\text { attitudes/negative thoughts regarding diabetes } \\
\text { - Activation of resources } \\
\text { - Mindfulness intervention } \\
\text { - Acceptance intervention (specifically regarding } \\
\text { diabetes acceptance and integration) } \\
\text { - Goal setting and attainment scaling } \\
\text { - Maintenance techniques }\end{array}$ & $\begin{array}{l}N=125 \text { ( } 95.4 \% \text { of those randomized to stepped care) } \\
\text { attended the group treatment. Of them, } n=77 \\
(61.6 \%) \text { attended all five group sessions, } n=43 \\
\text { (34.4\%) attended four sessions, and } n=5(4.0 \%) \\
\text { attended three sessions or less ( } 3 \text { sessions, } n=2 ; \\
2 \text { sessions, } n=2 ; 1 \text { session, } n=1) \\
\text { Reasons for non-participation in step } 1(n=6) \text { were: } \\
\text { - dropout at baseline ( } n=4) \\
\text { - decision to reject the treatment ( } n=1) \\
\text { - inability to attend for timing reasons }(n=1) \\
\text { Reasons for treatment discontinuation }(=\text { attending } \\
\text { less than four sessions) were: } \\
\text { - timing problems }(n=3) \\
\text { - decision to terminate the treatment }(n=2)\end{array}$ \\
\hline Step 3 & $\begin{array}{l}\text { Referral for outpatient depression } \\
\text { treatment by psychotherapist } \\
\text { and/or psychiatrist and/or AD } \\
\text { treatment monitored by a doctor }\end{array}$ & $\begin{array}{l}\text { Provided interventions were not pre-defined but } \\
\text { depended on the providers to whom participants } \\
\text { were referred. Referral was generally non- } \\
\text { mandatory, that is, participants decided } \\
\text { themselves upon following the study } \\
\text { psychologist's advice to see the doctor for referral } \\
\text { to outpatient depression treatment and/or } \\
\text { initiation of AD treatment. Received treatments } \\
\text { were documented according to subjects' reports } \\
\text { by the study psychologist }\end{array}$ & $\begin{array}{l}N=14 \text { ( } 10.7 \% \text { of those in stepped care) were referred } \\
\text { for outpatient depression treatment (due to } \\
\text { insufficient response after step } 2) \text {; however, } 1 \text { person } \\
\text { did not initiate further treatment. Of } 13 \text { patients } \\
\text { receiving treatment, } 8 \text { had PT ( } 1-14 \text {, mean } \pm \text { SD }= \\
6.0 \pm 4.3 \text {, sessions), four times combined with } A D(1 \\
\text { also saw a psychiatrist thrice), while five took } A D \\
\text { only (i.e., without PT, but } 1 \text { saw a psychiatrist twice), } \\
\text { and } 1 \text { saw a psychiatrist only ( } 4 \text { visits). Notably, } 6 \text { of } \\
\text { them additionally received inpatient mental health } \\
\text { treatment ( } 3-14 \text { weeks, mean } \pm S D=5.0 \pm 4.5 \text { ) at } \\
\text { some point, which was not a designated component } \\
\text { of the stepped care approach }\end{array}$ \\
\hline
\end{tabular}

$A D$, antidepressant; PT, psychotherapy.

manualized 5-session CBT group treatment program "DIAMOS - Strengthening Diabetes Motivation" was effective in reducing depressive symptoms and diabetes distress in patients with diabetes and subclinical depression [24]. Participants received five 90min sessions in small groups. The program consists of interventions aiming to help patients reduce mild depressive symptoms and elevated diabetes distress. We considered this a suitable lowintensity intervention for the initial treatment step, effective for people with subclinical clinical affective conditions and potentially beneficial also for those with mild-to-moderate clinical depression. The program's five sessions and corresponding interventions are described in Table 1 . Step 1 was provided during the inpatient phase at the diabetes center.
For the increased-intensity second treatment step we chose a brief single CBT intervention for major depression which was provided during the outpatient phase via telephone and came with a therapy guidebook as supporting material. Six weekly sessions of telephone-delivered CBT (45 min each) were mandatory, including explanatory models of the development and persistence of depressed mood through depressogenic cognitions, the detection of negative thought patterns, self-observation and stopping of negative thinking, prevention of catastrophic and harmful thought patterns, restructuring of negative cognitive schemas, training of more realistic thinking and positive self-instructions for the day (detailed in Table 1). Specific exercises regarding self-criticism, self-esteem, worry and fear, sleep problems, fatigue, work inability, 
social and relationship problems could be delivered based upon individual demand, with up to six additional sessions to be offered if required by the patient's progress or persistence.

For the final step 3 (i.e., in case of persistent non-response following the previous treatment steps), referral for outpatient depression treatment was selected (Table 1). That is, concerned patients were informed about their persistently elevated depressive symptoms despite previous treatment and the possible benefit of a more specialized/more intensive mental treatment; the patient was advised to see the doctor and discuss possible referral for standard outpatient depression treatment according to guidelines [27], that is, psychotherapy and/or psychiatric treatment and/or antidepressant treatment to be monitored by the doctor. The study psychologist supported the patient in undertaking the necessary steps for initiation of step 3. However, a patient's engagement in this step was voluntary and suggested treatments could be rejected. For participants receiving step 3 , the implemented treatments were documented according to patient reports by the study psychologist.

Full explanations of the interventions and effect factors of the stepped care approach are provided in online supplementary Table S1 (see www.karger.com/doi/10.1159/000520319 for all online suppl. material). Participants receiving stepped care were treated until a meaningful depression reduction was achieved or all steps were passed. Meaningful reduction was defined as a decrease of the Hamilton Rating Scale for Depression (HAMD) severity score below the clinical level (score under 9 ) or by $\geq 50 \%$ compared to baseline; if the score was below 9 at baseline, $a \geq 50 \%$ reduction was required.

Diabetes care as usual at the diabetes center included education and treatment provided by the center's diabetologists, diabetes nurses and nutritionists, evaluation, adaption and dose-adjusting of medical treatments, structured glucose monitoring and selfmanagement practice and educational lessons regarding acute and long-term complications, sports and activities, foods, cooking and recipes, and social aspects of diabetes. Diabetes care as usual was provided to participants in both study groups equivalently.

\section{Measurement Time Points and Outcomes}

Outcomes were assessed at baseline, 6- and 12-month FU; some core aspects also at the end of the inpatient phase ("posttest"). Depression severity was monitored at the end of the inpatient phase (in-person interview) as well as 3 and 6 months after it (telephone interview) to evaluate depression reduction compared to baseline and decide upon further steps. Primary outcome was the rate of meaningful depression reduction at 12-month FU according to HAMD ( $<9$ or by $\geq 50 \%$ ). Changes in depressive symptoms, diabetes distress, diabetes acceptance, anxiety, well-being, quality of life, self-care, treatment satisfaction, $\mathrm{HbA}_{1 \mathrm{c}}$, and biomarkers of inflammation were secondary outcomes.

\section{Primary Outcome}

Depression severity was assessed using the semi-structured 17item HAMD interview by trained psychologists. The GRID version (requiring separate symptom severity and frequency ratings using a standardized system) with higher reliability and good clinimetric properties was used [30-32]. The total score ranges from 0 to 52 . Scores $<9$ indicate no, 9-16 mild, $17-24$ moderate, and $>24$ severe depression [33].

Stepped Care Approach for Depression and Distress in Diabetes

\section{Secondary, Patient-Reported Outcomes}

The CES-D [34], used for screening, was included as a secondary, self-report-based measurement of depressive symptoms. Furthermore, several patient-reported outcome measures were used to capture the patients' perceptions of their health, well-being, mental symptoms, and diabetes-related aspects (described in online suppl. Table S2): diabetes distress was assessed with the PAID [35]. Anxiety was assessed using the State-Trait Anxiety Inventory (STAI) [36]. Emotional well-being was measured using the WHOFive Well-being Index (WHO-5) [37]. Diabetes acceptance was measured using the 6-item Acceptance and Action Diabetes Questionnaire (AADQ) [38]. Health-related quality of life was assessed by the EuroQol Five-Dimensions Health Questionnaire (EQ-5D) and the 36-Item Short Form Health Survey (SF-36) [39]. Diabetes self-care was assessed by the 10-item Summary of Diabetes SelfCare Activities Measure (SDSCA) [40]. Furthermore, the 16-item Diabetes Self-Management Questionnaire (DSMQ) [41] was administered. Treatment satisfaction was assessed using the Diabetes Treatment Satisfaction Questionnaire (DTSQ) [42].

\section{Secondary, Laboratory-Assessed Outcomes}

Glycated hemoglobin $\left(\mathrm{HbA}_{1 \mathrm{c}}\right)$ was estimated from venous blood samples, taken at the same time as the psychological assessments, in the central laboratory by HPLC (Tosoh Automated Glycohemoglobin Analyzer HLC-723G11; IFCC standard; normal range: $23.5-43.2 \mathrm{mmol} / \mathrm{mol}$; $4.3-6.1 \%$ ).

Selected biomarkers of inflammation and adipocyte function were estimated from cryopreserved serum samples, collected at baseline and 12-month FU. Baseline and FU samples of each participant were analyzed in the same assay to avoid bias due to interassay variability. Biomarkers, chosen for associations with depression, glycemia, and complications [14-19], were: high-sensitivity C-reactive protein (hs-CRP), determined using a Roche/Hitachi cobas c311 Analyzer (Basel, Switzerland); interleukin (IL)-1Ra, IL6, CC-chemokine ligand 2 (CCL2; also known as monocyte chemotactic protein-1/MCP-1), and total adiponectin, estimated using Quantikine (for IL-1Ra, CCL2, and adiponectin) or Quantikine HS (for IL-6) ELISA kits (R\&D Systems, Wiesbaden, Germany), and IL-18, estimated using an MBL ELISA kit (Nagoya, Japan).

\section{Descriptive Personal and Diabetes-Related Variables and Covariates}

The demographic and diabetes-related characteristics collected for this study comprised sex, age, BMI, years of education, diabetes type, diabetes duration, diabetes treatment and diagnosed diabetes complications, according to patient files. Data on complications of diabetes were based on medical examinations, laboratory assessments, and information from files: diagnoses of coronary heart disease, peripheral artery disease, and stroke were based on a previous events or revascularization measures; diabetic nephropathy was based on a glomerular filtration rate below $60 \mathrm{~mL} / \mathrm{min} / 1.73$ $\mathrm{m}^{2}$ and/or persistent albuminuria according to international guidelines; diabetic retinopathy was based on ophthalmologic examination or previous laser coagulation treatment; diabetic neuropathy was based on the neuropathy deficit score.

Participants were asked to report possible mental treatment during the past year (i.e., finished or discontinued as current treatment was excluded) to evaluate the recent history of mental treatment. Current medications affecting biomarkers of inflammation 


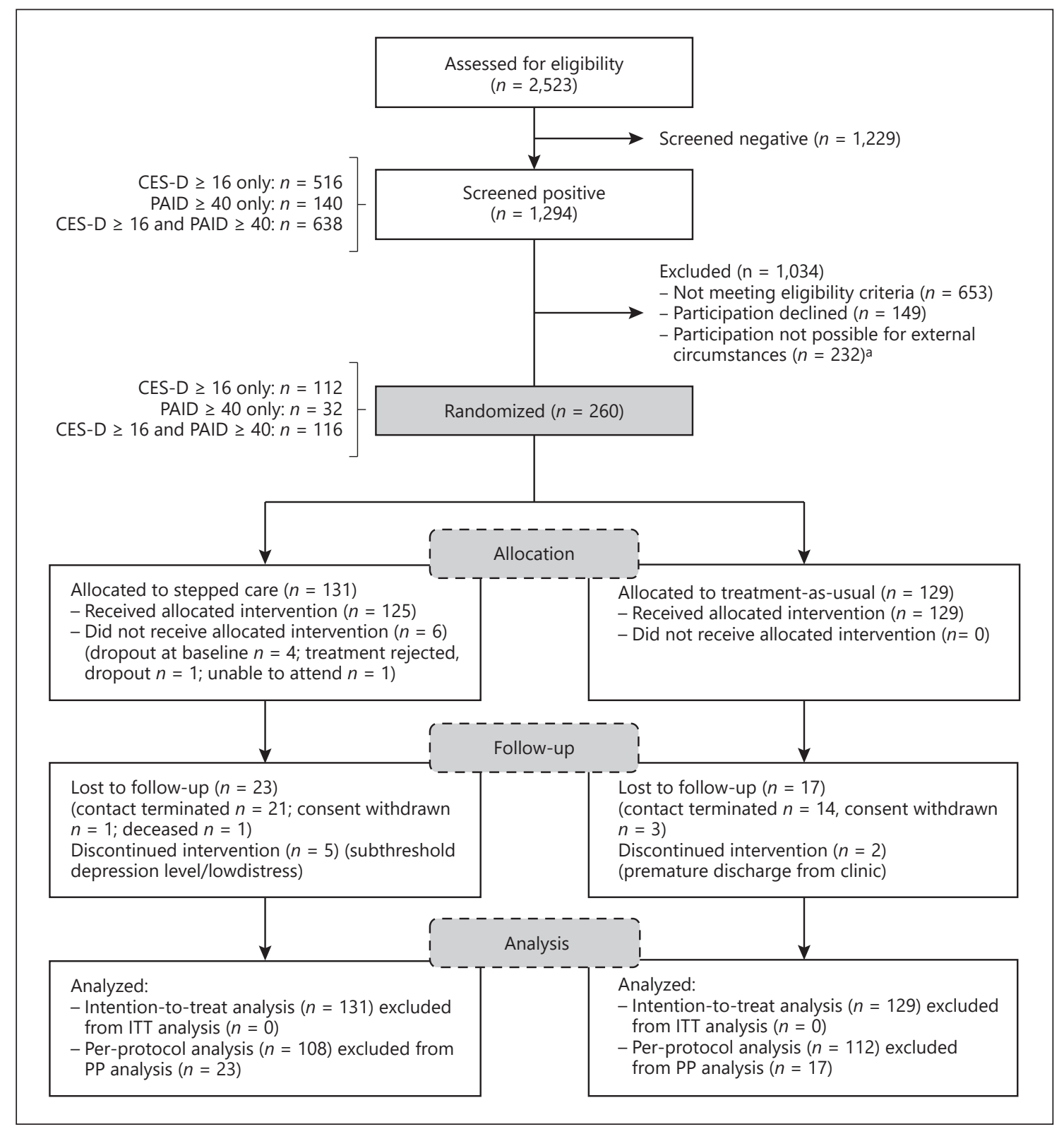

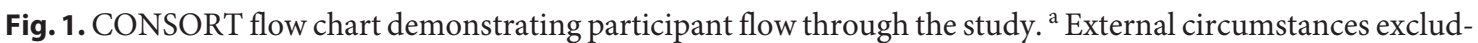
ing participation $(n=232)$ were organizational and/or timing problems in the context of the inpatient stay at the recruiting study center hindering a person's treatment attendance.

(i.e., statins, anticoagulants, thyroid medication, corticosteroids, and non-steroidal anti-inflammatory drugs) were documented as covariates.

\section{Sample Size}

Expected depression reduction rates were $>70 \%$ for the treatment group versus $<50 \%$ for the control group. Overall, 192 participants (96 per group) were required for testing treatment superiority with a two-sided error probability $(\alpha)$ of 0.05 and statistical power $(1-\beta)$ of 0.85 ( $G^{*}$ Power 3.1). Based on an expected non- evaluable rate of $25 \%$, a total of $(192 * 4 / 3=) 256$ participants were needed.

\section{Statistical Analyses}

The analyses were performed using SPSS 24.0.0 (IBM SPSS Statistics). The primary outcome was tested using Pearson's $\chi^{2}$ test. An intention-to-treat (ITT) approach was used with missing HAMD values at the 12-month FU replaced using automatic multiple imputation (MI; SPSS 24 missing data module; 10 estimations per missing value with predictors: age, sex, education years, diabe- 
Table 2. Baseline characteristics of the study groups

Stepped care group

$(n=131)$

\section{Demographic and diabetes-related variables}

Age, years

Female sex

Years of education, years

BMI

Type 1 diabetes

Diabetes duration, years

With insulin

$\mathrm{HbA}_{1 \mathrm{c}}, \mathrm{mmol} / \mathrm{mol}$

$\mathrm{HbA}_{1 \mathrm{c}} \%$

With microvascular complications ${ }^{a}$

With macrovascular complications ${ }^{\mathrm{b}}$

Psychological variables/patient-reported outcomes

HAMD score (0-52) - depression severity

CES-D score (0-60) - depressive symptoms

PAID score (0-100) - diabetes distress

AADQ score (6-30) - diabetes acceptance

WHO-5 score (0-25) - emotional well-being

STAI-S score (0-80) - state anxiety

STAI-T score (0-80) - trait anxiety

EQ-5D TTO score (0-1) - five dimensions health state score

EQ-5D VAS score (0-100) - overall health state

SF-36 PCS $(50 \pm 10)$ - physical health state

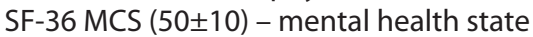

DTSQ score (0-6) - treatment satisfaction

SDSCA total score (0-7) - self-care activities

DSMQ total score (0-10) - diabetes self-management

\section{Markers of inflammation ${ }^{c}$}

hs-CRP, $\mathrm{mg} / \mathrm{dL}$

IL-6, pg/mL

IL-18, pg/mL

IL-1RA, pg/mL

$\mathrm{CCL} 2, \mathrm{pg} / \mathrm{mL}$

Adiponectin, $\mathrm{ng} / \mathrm{mL}$
Treatment-as-usual group $\quad p$ value

$(n=129)$

$45.2 \pm 13.9$
$75(57.3)$
$12.3 \pm 2.3$
$29.8 \pm 6.9$
$81(61.8)$
$14.6 \pm 10.6$
$117(89.3)$
$72 \pm 17$
$8.7 \pm 1.5$
$50(38.2)$
$15(11.5)$

$44.9 \pm 15.1$

67 (51.9)

0.881

$12.2 \pm 2.2$

$29.7 \pm 7.3$

$85(65.9)$

$14.3 \pm 10.0$

120 (93.0)

$73 \pm 17$

$8.8 \pm 1.5$

49 (38.0)

15 (11.6)

0.390

0.568

0.968

0.496

0.822

0.292

0.611

0.611

0.976

0.964

$\begin{array}{ll}11.0 \pm 4.9 & 0.384 \\ 23.8 \pm 9.8 & 0.989 \\ 39.8 \pm 18.4 & 0.961 \\ 14.9 \pm 5.0 & 0.451 \\ 9.8 \pm 5.4 & 0.293 \\ 44.6 \pm 10.9 & 0.201 \\ 48.8 \pm 10.5 & 0.305 \\ 0.83 \pm 0.21 & 0.323 \\ 62.3 \pm 18.4 & 0.712 \\ 45.7 \pm 10.6 & 0.840 \\ 35.7 \pm 11.1 & 0.508 \\ 22.1 \pm 6.8 & 0.747 \\ 3.3 \pm 1.2 & 0.637 \\ 6.0 \pm 2.1 & 0.582 \\ & \\ 0.15(0.06 ; 0.31) & 0.786 \\ 1.31(0.86 ; 2.26) & 0.926 \\ 217.9(173.8 ; 274.4) & 0.784 \\ 437.2(308.9 ; 699.1) & 0.974 \\ 694.1(582.0 ; 921.8) & 0.785 \\ 8,234.0(4,133.0 ; 11,541.8) & 0.977\end{array}$

Data are presented as the mean \pm SD or $n(\%)$, or median (IQR) for markers of inflammation. Between-group comparisons: Student's $t$-test or Pearson's $X^{2}$ test. BMI, body mass index; $\mathrm{HbA}_{1 c}$, glycated hemoglobin; HAMD, Hamilton Rating Scale for Depression; CES-D, Center for Epidemiologic Studies Depression Scale; PAID, Problem Areas in Diabetes Scale; AADQ, Acceptance and Action Diabetes Questionnaire; WHO-5, WHO-Five Well-Being Index; STAI, State-Trait Anxiety Inventory; EQ-5D, EuroQol Five Dimensions Health Questionnaire; TTO, time trade off; VAS, visual analogue scale; SF-36, 36-Item Short Form Health Survey; PCS, physical component score; MCS, mental component score; SDSCA, Summary of Diabetes Self-Care Activities Measure; DSMQ, Diabetes Self-Management Questionnaire; DTSQ, Diabetes Treatment Satisfaction Questionnaire; hs-CRP, high-sensitivity C-reactive protein; IL, interleukin; IL-1RA, IL-1 receptor antagonist; CCL2, CCchemokine ligand 2; IQR, interquartile range. ${ }^{a}$ Diabetic retinopathy, neuropathy, nephropathy. ${ }^{b}$ Peripheral artery disease, coronary heart disease, stroke. ${ }^{c}$ Statistical tests of markers of inflammation were performed based on In-transformed values due to skewed raw score distributions.

tes type, duration, available previous HAMD scores). For sensitivity analysis, an ITT analysis using the last observation carried forward (LOCF) method was added. A per-protocol analysis was performed using the study completers (i.e., those lacking HAMD data at FU were excluded).
Secondary outcomes were analyzed using repeated-measures ANOVA with group as the independent and baseline-to-FU change as the dependent variable. Paired samples $t$-tests were used for testing within-group baseline-to-FU changes.

Biomarkers of subclinical inflammation were analyzed based on ln-transformed values for skewed distributions, while in the 
descriptive statistics median raw scores and interquartile ranges are reported for interpretability. These analyses were adjusted for: age, sex, diabetes type, duration, microvascular/macrovascular complications, and inflammation-relevant medications (above) as recommended [15]. $p$ values $<0.05$ (two-tailed significance) were considered to indicate statistical significance in all analyses.

\section{Results}

\section{Participant Enrolment}

Of a total of 2,523 patients with diabetes screened using the CES-D and PAID, 1,294 (51.3\%) screened positive (Fig. 1). Thereof, 641 (49.5\%) were eligible based on inclusion/exclusion criteria; however, 232 persons could not participate for external conditions (e.g., time/organizational issues). Of 409 subjects eligible and invited, 260 (63.6\%) consented and were randomized: 131 to stepped care, 129 to treatment-as-usual.

\section{Sample Characteristics}

The full sample characteristics by group are given in Table 2. Fifty-five percent were women; the mean age was $45.0( \pm 14.5)$ years. Sixty-four percent had type 1 diabetes (in line with the recruiting center's patient population). The mean diabetes duration was $14.4( \pm 10.3)$ years. Most $(91.2 \%)$ used insulin. The mean glycemic levels were above target ranges $\left(\mathrm{HbA}_{1 \mathrm{c}}: 72 \pm 17 \mathrm{mmol} / \mathrm{mol} ; 8.8 \pm\right.$ $1.5 \%)$. Thirty-eight percent had microvascular and $11.5 \%$ had macrovascular complications.

The mean baseline CES-D and PAID scores were 23.8 $( \pm 9.6)$ and $39.9( \pm 18.5)$, respectively, reflecting elevated depressive symptoms and diabetes distress. The baseline HAMD score was $10.7( \pm 4.7)$, with $65.4 \%$ scoring above 9. There were no significant differences between groups at baseline (Table 2).

Twenty-one participants $(8.1 \%)$ reported having had mental treatment during the past year: 12 had seen a psychologist, 2 had seen a psychiatrist, 4 had seen both, and 3 had taken antidepressants. There were no significant differences in terms of past mental treatment between the study groups.

\section{Dropout}

Forty subjects (15.4\%) were lost to FU; these were younger, had a lower education level, shorter diabetes duration, and higher $\mathrm{HbA}_{1 c}$; they also reported less optimal self-care and higher anxiety (online suppl. Table S3).

\section{Received Interventions}

Table 1 shows the provided stepped care interventions (sequence shown in online suppl. Fig. S1). Of those receiving stepped care, 125 (95.4\%) attended the CBT group treatment (step 1). Forty of them (32.0\%) did not yield sufficient depression reduction, thus receiving single CBT (step 2) consequently with an average of 7 sessions per person. Ultimately, 14 of them (35.0\%) were referred for outpatient depression treatment (step 3). Eight subjects received psychotherapy, 4 of them combined with antidepressants; 5 received antidepressants; 1 did not initiate further treatment. Notably, 6 subjects ultimately received inpatient mental health treatment (i.e., no designated part of the stepped care approach). Additionally, 5 participants of the stepped care group reported having had mental health treatment (4 antidepressants, 1 psychotherapy) during the study without referral and despite non-elevated HAMD depression levels.

Of those in the treatment-as-usual control group, 127 (98.4\%) completed the treatment and 2 discontinued. At the 12-month FU, 22 subjects (17.1\%) reported having had (self-initiated) mental health treatment during the year: 18 had had psychotherapy (1-41 sessions, mean \pm $\mathrm{SD}=10.9 \pm 11.6$ ), seven times combined with antidepressants, and 4 had taken antidepressants only; 3 of them had had inpatient mental health treatment ( $4-8$ weeks, mean $\pm \mathrm{SD}=6.0 \pm 2.0$ ).

\section{Effect on Primary Outcome}

ITT analysis: meaningful baseline-to-FU depression reduction was observed in 105 patients (80.2\%) in stepped care versus 66 patients $(51.2 \%)$ in treatment-as-usual ( $p$ $<0.001 ; 3.8$ vs. $16.3 \%$ reported significant deterioration, 16.0 vs. $40.3 \%$ remained unchanged; online suppl. Table S4); the sensitivity analysis yielded consistent results (Table 3 ). The per-protocol analysis indicated 87.0 versus $48.2 \%$ depression reduction in the treatment versus control group $(p<0.001)$. Depression reduction rates and depression score changes over the full study period are shown in Figure 2.

\section{Effect on Secondary Outcomes}

The full baseline-to-FU changes are shown in Table 3. The treatment group yielded a greater reduction in depression severity scores (HAMD) than the control group $(\Delta-3.2, p<0.001$; Fig. 2). However, the reduction of depressive symptoms according to the CES-D was not significantly greater in the treatment group than the control group $(\Delta-2.3, p=0.065)$. The reduction of diabetes distress was also not significantly greater in the treatment 


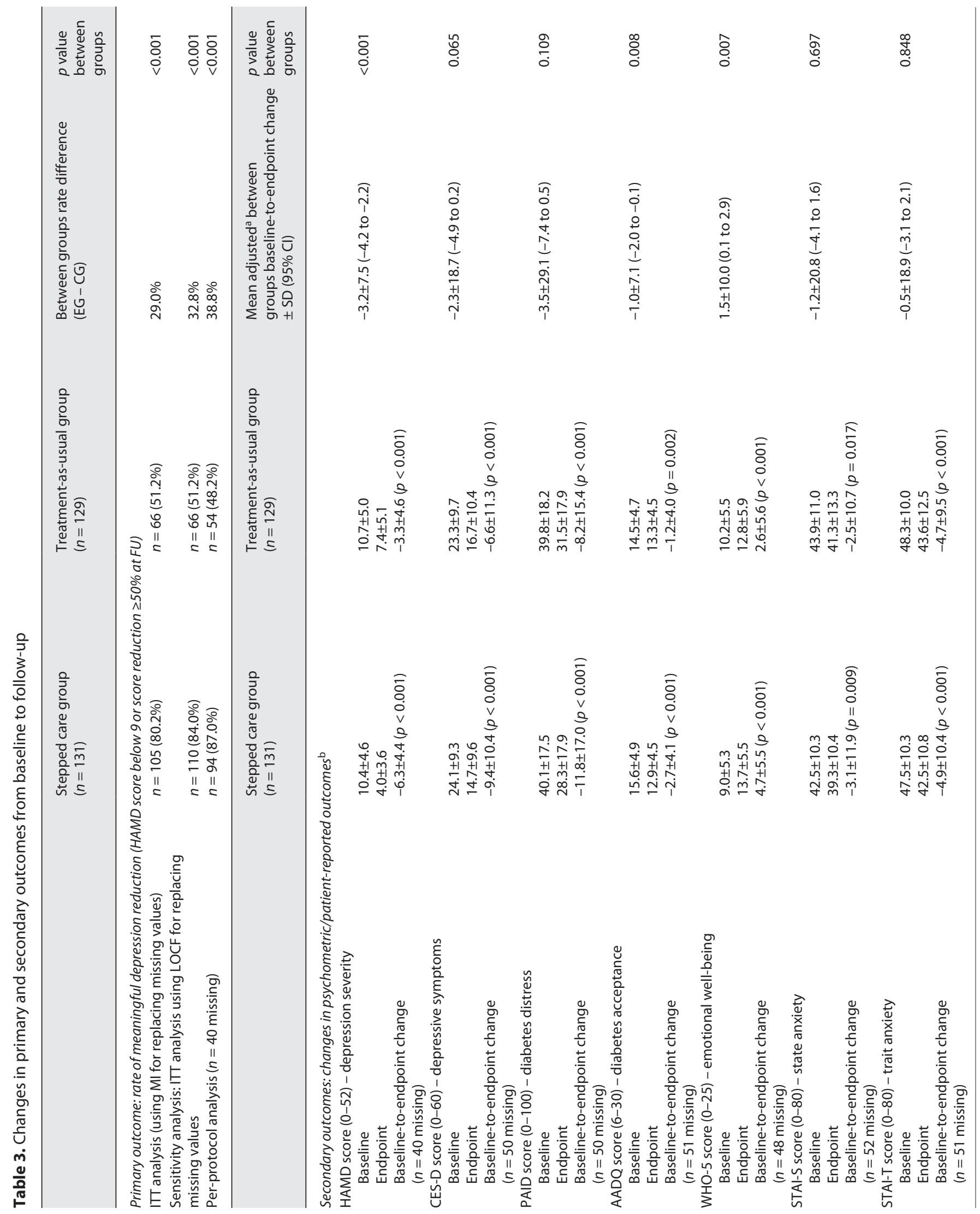




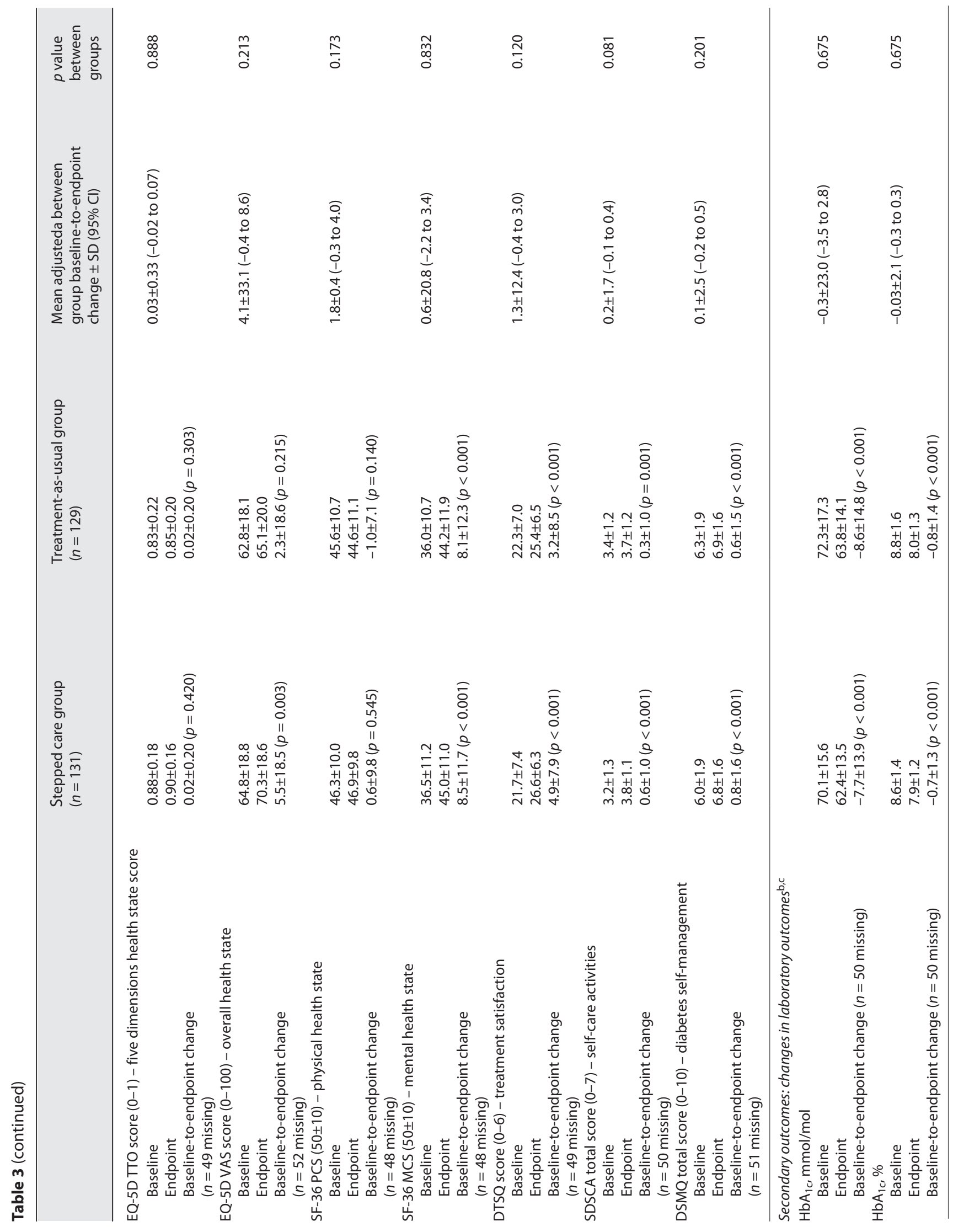




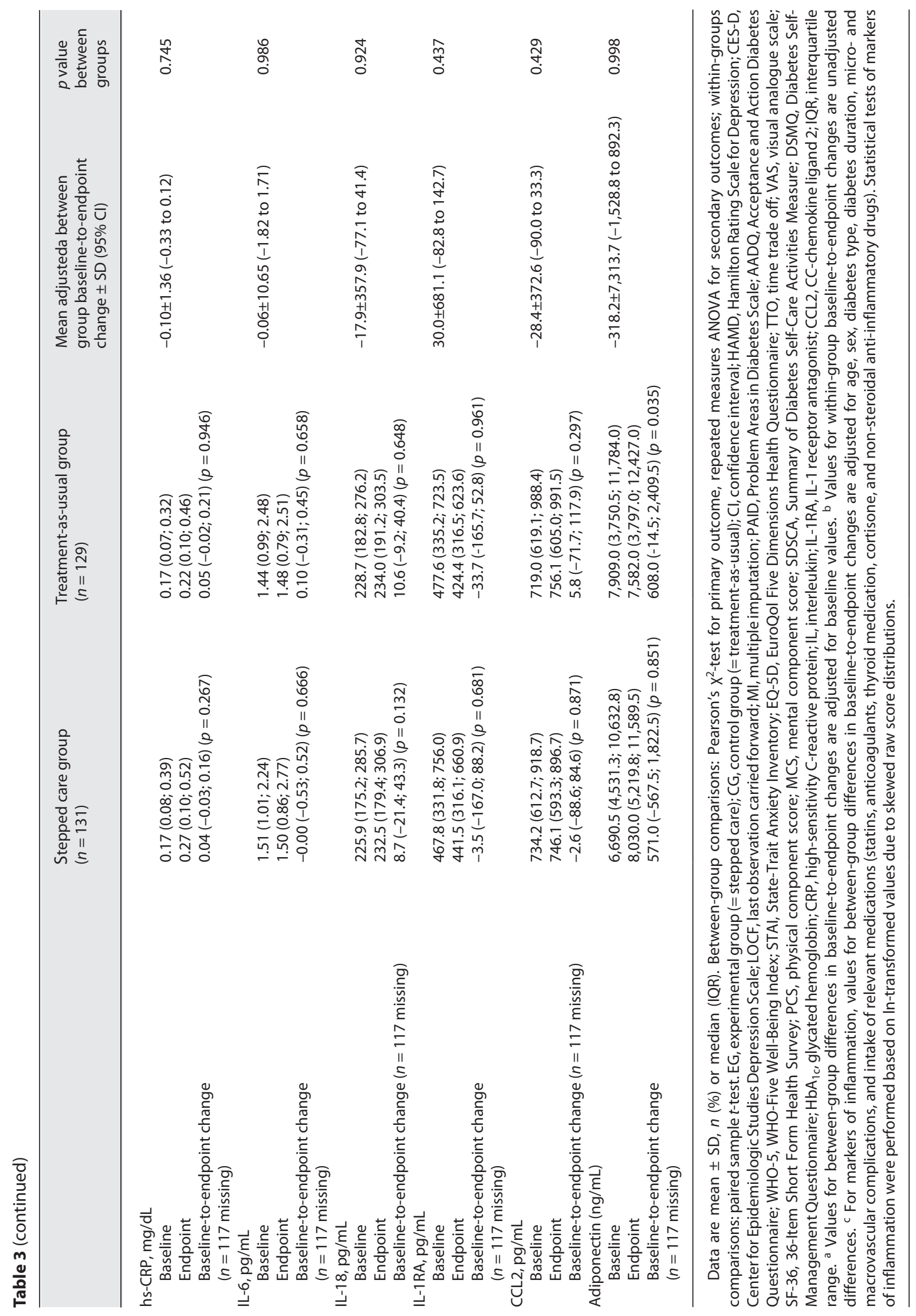




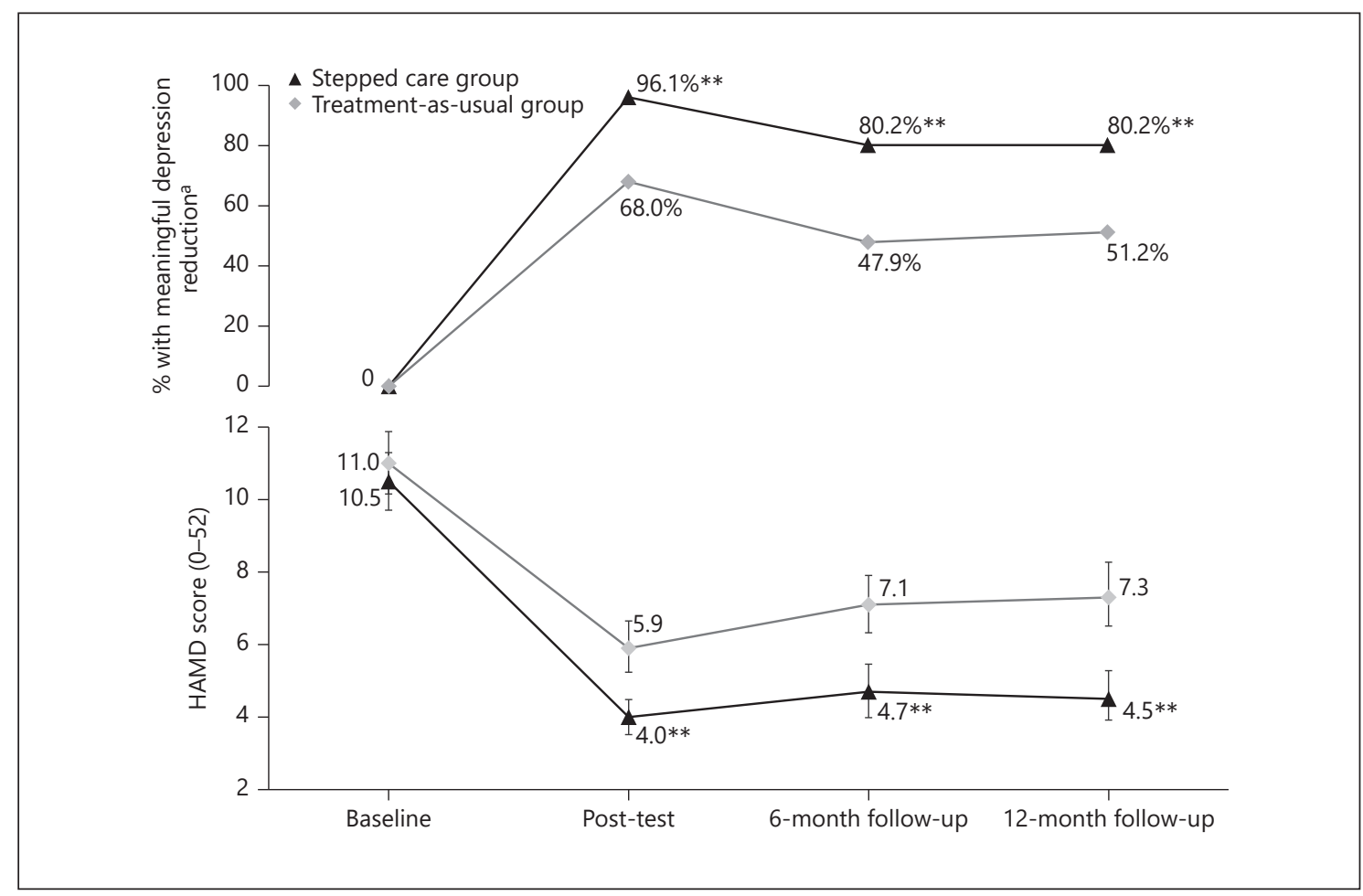

Fig. 2. Depression reduction rates and HAMD score changes over the follow-up period by group (ITT population). Data are \% scores or the mean and 95\% CI (error bars). Between-group comparisons: Pearson's $\chi^{2}$ test for frequencies, repeated measures ANOVA for depression scores. HAMD, Hamilton Rating Scale for Depression; $\mathrm{CI}$, confidence interval. ${ }^{\text {a }}$ Meaningful depression reduction is defined as an HAMD score $<9$ or HAMD score reduction $\geq 50 \%$ at FU. ${ }^{* *} p<0.001$. Missing values: post-treatment, $n=4$; 6-month follow-up, $n=25 ; 12$-month $\mathrm{FU}$, missing values replaced (MI).

group $(\Delta-3.5, p=0.109)$. However, the treatment group displayed greater improvements in emotional well-being $(\Delta 1.5, p=0.007)$ and diabetes acceptance $(\Delta-1.0, p=$ $0.008)$. Regarding anxiety, quality of life, and treatment satisfaction both groups showed significant improvements but no difference between groups (all $p \geq 0.120$ ). There was a tendency towards a greater improvement in self-care according to SDSCA following stepped care $(\Delta 0.20, p=0.081)$; however, changes in DSMQ scores did not suggest different self-management $(p=0.201)$. $\mathrm{HbA}_{1 c}$ improved in both groups without between-group difference $(p=0.675)$.

No significant changes between baseline and FU levels of biomarkers of subclinical inflammation were observed in the fully adjusted analyses, neither within nor between groups (Table 3). Furthermore, adding baseline levels of biomarkers of subclinical inflammation to the analyses of depression levels as covariates did not alter the findings, nor did it indicate interactions, suggesting that inflammatory pathways did not modulate the depression treatment response.

\section{Discussion}

Mental healthcare for patients with diabetes and elevated depression symptoms as well as those with diabetes distress is recommended by current practice guidelines $[25,26]$. This randomized trial observed a greater rate of depression reduction in patients with diabetes receiving stepped care for depression and diabetes distress compared to treatment-as-usual. Stepped care was effective in treating comorbid depression in type 1 diabetes and type 2 diabetes. The intervention had further beneficial effects on illness acceptance and well-being.

The stepped care approach included a diabetes-specific group treatment for patients with diabetes distress and depressive symptoms as the first step. The treatment, focusing on diabetes-related burdens and acceptance, was found to reduce depressive symptoms and prevent major depression in a previous randomized trial [24]. In the present study, the majority of participants responded and reached a sufficient depression reduction right after this 
initial treatment step, suggesting that a brief diabetes-oriented CBT intervention may be useful for a majority of patients with diabetes and depressive symptoms on the short term. This inference is corroborated by the finding that diabetes acceptance was improved simultaneously, whereby low acceptance has been associated with high diabetes distress and depressive symptoms [38].

However, in about one third of these participants the initial effect diminished with more intensive and specific depression treatment subsequently required. Still, the treatment response to a brief diabetes-specific intervention supports the putative relevance of diabetes distress for increasing and maintaining depressive symptoms, suggesting that these aspects need to be addressed. A brief CBT-based group intervention aiming at the reduction of diabetes distress and depressive symptoms may constitute a starting point for treating patients with diabetes with (non-severe) clinical depression while avoiding unnecessary side-effects of antidepressants and saving resources compared to standard psychotherapy.

Improvements in CES-D depressive symptoms scores were less pronounced than for HAMD depression scores which might be explained by differential sensitivity of the two rating scales [43] and/or differences of the assessed symptoms: the HAMD covers the somatic symptoms of depression (e.g., psychomotor agitation/retardation, sleep problems, weight loss, libido change) better than the CES$\mathrm{D}$; it also requests suicidal ideation. The CES-D includes items regarding interpersonal problems as well as four positively keyed items which may confuse respondents and yield bias [34]. Another issue yielding divergence is the non-availability of CES-D scores in some participants whose HAMD scores were evaluated: 10 participants completing the HAMD interview never returned the CES-D questionnaire. Finally, questionnaires are associated with response bias, while the semi-structured interview may limit bias due to discriminatory questioning. After all, the standardized GRID version of the HAMD used here is a gold standard measurement of depression severity [32] meeting criteria for a valid and sensitive clinimetric tool [43].

Changes in diabetes distress, anxiety, and health-related quality of life scores following the treatment were comparable across groups: both groups reported improvements without differences between the groups. The same was true for the treatment-related measures of self-care, glycemic outcome, and treatment satisfaction. This may be partly explained by the active control condition including diabetes education and care at a specialized inpatient center. Also, specialized diabetes care may have beneficial effects on diabetes distress and mood problems, and could have im- proved these aspects in the control group. The positive impact of the control condition is also supported by the finding of improved treatment satisfaction in this group.

The results regarding glycemic outcome appear in line with previous trials of psychological and pharmacological depression treatment in patients with diabetes which were effective in reducing depressive symptoms, while effects on $\mathrm{HbA}_{1 \mathrm{c}}$ were either small or lacking [44]. This suggests that improving depressive symptoms does not automatically lead to better glycemia in diabetes.

When evaluating the observed improvements of mental health outcomes in this study, the number of control participants who initiated depression treatment themselves within the follow-up period should be considered, suggesting that effect estimations between groups might be conservative. Notably, more participants of the control group than the treatment group initiated specialized mental health treatments which might point to a positive effect of stepped care.

Few studies have analyzed possible effects of CBTbased depression treatment on subclinical inflammation in patients with diabetes. The present study found no significant changes in biomarkers of subclinical inflammation between baseline and FU in either of the groups, and accordingly no differences between groups. However, the sample size was relatively low for these analyses and therefore combined patients with type 1 diabetes and type 2 diabetes. In a previous study combining biomarker datasets from the ECCE HOMO and DIAMOS studies [15], analyses stratified by diabetes type showed that reductions in depressive symptoms were associated with reductions in biomarkers of inflammation in patients with type 2 diabetes but not type 1 diabetes. There is also evidence from other cohorts that type 1 diabetes and type 2 diabetes may differ in this respect [14], which should be considered when interpreting our data.

\section{Limitations and Strengths of the Study}

Several limitations must be considered: First, the study sample was a mixed patient sample collected at a specialized diabetes center which resulted in an overrepresentation of patients with type 1 diabetes and insulin-treated type 2 diabetes compared to primary care. Although depressive symptoms and diabetes distress are common comorbid conditions for both types of diabetes, specific requirements and burdens may vary with treatment regimens, thus there is a risk of heterogeneity due to the inclusion of both diabetes types; yet changes in outcomes were comparable across diabetes types. Second, patients with severe major depression (F32.2) were excluded, thus 
generalization of the results may be limited to non-severe forms of depression (F32.0, F32.1, elevated depressive symptoms). Third, despite rather confined dropout rates, analyses regarding primary and secondary outcomes included partly different case numbers, and the analyses regarding markers of inflammation were limited by significant missing data. Finally, although patients receiving external psychological or pharmacological treatment for depression at the time of eligibility screening were excluded from participation, there were no criteria defining eligibility according to previous mental treatments, thus patients were included and randomized irrespective of a potential history of depression treatment. Similarly, patients were enrolled according to current depressive symptoms and/or diabetes distress only, that is, irrespective of possible earlier stages of depressive symptoms or disorders (e.g., recurrent, persistent, remitted).

The strengths of this study are the evaluation of a stepped care approach using a randomized controlled study design supporting reliable inferences for clinical diabetes care (e.g., graduated treatment for patients with diabetes and depressive symptoms); and the data collection using validated measures, temporal coincidence of psychological and $\mathrm{HbA}_{1 \mathrm{c}}$ assessment, consideration of the relevance of subclinical inflammation, and multivariate adjustment of all analyses.

\section{Conclusion}

The findings support that the stepwise increase of treatment intensity based on individual demand constitutes an effective approach for tailoring treatment of comorbid depression in diabetes. The inclusion of interventions for diabetes distress appears beneficial regarding the prevalent group with mild-to-moderate depressive symptoms. The approach did not yield greater improvement in diabetes distress than specialized diabetes care, which suggests that diabetes education and support regarding diabetes problems can reduce diabetes-related distress. Regarding patients with concomitant depression and diabetes distress, treatments focusing on the interaction between mood and diabetes-related issues have been recommended. Furthermore, it has been suggested that concerned patients are more likely to benefit from disease-specific treatments than standard depression therapy. Our results seem to correspond to these suggestions. In summary, brief CBT-based programs regarding diabetes-related problems (e.g., regarding the bidirectional interaction between mood, diabetes and health) constitute a starting point for treating patients with diabetes with affective symptoms, with specialized depression treatment to be offered upon demand.

\section{Acknowledgements}

The authors would like to thank Ulrike Partke, German Diabetes Center (DDZ) Duesseldorf, and Silke Balbach, Linda Geißendörfer, and Thomas Weber, Diabetes Center Mergentheim, for excellent laboratory assistance.

\section{Statement of Ethics}

Study approval: the study protocol was reviewed and approved by the Ethics Committee of the State Medical Chamber of BadenWuerttemberg, approval number F-2013-011.

Consent to participate: all study participants provided written informed consent prior to inclusion.

\section{Conflict of Interest Statement}

The authors have no conflicts of interest to declare.

\section{Funding Sources}

This work was supported by the Competence Network for Diabetes mellitus, grant No. 01GI1107, and the German Center for Diabetes Research (DZD), grant No. 82DZD01101, funded by the Federal Ministry of Education and Research. The German Diabetes Center (DDZ) Duesseldorf is supported by the Ministry of Culture and Science of the State of North Rhine-Westphalia and the German Federal Ministry of Health. The funders were not involved in the design of the study, the collection, analysis, and interpretation of data, writing the report, and did not impose any restrictions regarding the publication of the report.

\section{Author Contributions}

A.S. co-designed the study, collected the data, researched the data, and wrote the manuscript. B.K. and N.H. designed the study, discussed the results, and reviewed/edited the manuscript. A.R. collected the data. C.H. analyzed biomarkers of inflammation, discussed the results, and reviewed/edited the manuscript. M.R. and T.H. discussed the results and reviewed/edited the manuscript. A.S. is the guarantor taking responsibility for the contents of the article.

\section{Data Availability Statement}

The data that support the findings of this study are not publicly available due to data protection regulations but are available from the corresponding authors (A.S.) upon reasonable request. 


\section{References}

1 Barnard KD, Skinner TC, Peveler R. The prevalence of co-morbid depression in adults with Type 1 diabetes: systematic literature review. Diabet Med. 2006 Apr;23(4):445-8.

2 Anderson RJ, Freedland KE, Clouse RE, Lustman PJ. The prevalence of comorbid depression in adults with diabetes: a meta-analysis. Diabetes Care. 2001 Jun;24(6):1069-78.

3 Perrin NE, Davies MJ, Robertson N, Snoek FJ, Khunti K. The prevalence of diabetes-specific emotional distress in people with type 2 diabetes: a systematic review and meta-analysis. Diabet Med. 2017 Nov;34(11):1508-20.

4 Fisher L, Gonzalez JS, Polonsky WH. The confusing tale of depression and distress in patients with diabetes: a call for greater clarity and precision. Diabet Med. 2014 Jul;31(7): 764-72.

5 Ehrmann D, Kulzer B, Haak T, Hermanns N. Longitudinal relationship of diabetes-related distress and depressive symptoms: analysing incidence and persistence. Diabet Med. 2015 Oct;32(10):1264-71.

6 Burns RJ, Deschênes SS, Schmitz N. Cyclical relationship between depressive symptoms and diabetes distress in people with type $2 \mathrm{di}$ abetes mellitus: results from the Montreal Evaluation of Diabetes Treatment Cohort Study. Diabet Med. 2015 Oct;32(10):1272-8.

7 Gonzalez JS, Peyrot M, McCarl LA, Collins EM, Serpa L, Mimiaga MJ, et al. Depression and diabetes treatment nonadherence: a meta-analysis. Diabetes Care. 2008 Dec;31(12): 2398-403.

8 Schmitt A, Reimer A, Hermanns N, Kulzer B, Ehrmann D, Krichbaum M, et al. Depression is linked to hyperglycaemia via suboptimal diabetes self-management: a cross-sectional mediation analysis. J Psychosom Res. 2017 Mar;94:17-23.

9 Lustman PJ, Anderson RJ, Freedland KE, de Groot M, Carney RM, Clouse RE. Depression and poor glycemic control: a meta-analytic review of the literature. Diabetes Care. 2000 Jul;23(7):934-42.

10 Nouwen A, Adriaanse MC, van Dam K, Iversen MM, Viechtbauer W, Peyrot M, et al. Longitudinal associations between depression and diabetes complications: a systematic review and meta-analysis. Diabet Med. 2019 Dec;36(12):1562-72.

11 Farooqi A, Khunti K, Abner S, Gillies C, Morriss $\mathrm{R}$, Seidu S. Comorbid depression and risk of cardiac events and cardiac mortality in people with diabetes: a systematic review and meta-analysis. Diabetes Res Clin Pract. 2019 Oct; 156:107816

12 Katon WJ, Rutter C, Simon G, Lin EH, Ludman E, Ciechanowski $P$, et al. The association of comorbid depression with mortality in patients with type 2 diabetes. Diabetes Care. 2005 Nov;28(11):2668-72.
13 Moussavi S, Chatterji S, Verdes E, Tandon A, Patel V, Ustun B. Depression, chronic diseases, and decrements in health: results from the World Health Surveys. Lancet. 2007 Sep; 370(9590):851-8.

14 Herder C, Hermanns N. Subclinical inflammation and depressive symptoms in patients with type 1 and type 2 diabetes. Semin Immunopathol. 2019 Jul;41(4):477-89.

15 Herder C, Schmitt A, Budden F, Reimer A, Kulzer B, Roden M, et al. Longitudinal associations between biomarkers of inflammation and changes in depressive symptoms in patients with type 1 and type 2 diabetes. Psychoneuroendocrinology. 2018 May;91:216-25.

16 Gohel MG, Chacko AN. Serum GGT activity and hsCRP level in patients with type 2 diabetes mellitus with good and poor glycaemic control: an evidence linking oxidative stress, inflammation and glycaemic control. J Diabetes Metab Disord. 2013 Dec; 12:56.

17 Moulton CD, Pickup JC, Ismail K. The link between depression and diabetes: the search for shared mechanisms. Lancet Diabetes Endocrinol. 2015 Jun;3(6):461-71.

18 Ismail K, Moulton CD, Winkley K, Pickup JC, Thomas SM, Sherwood RA, et al. The association of depressive symptoms and diabetes distress with glycaemic control and diabetes complications over 2 years in newly diagnosed type 2 diabetes: a prospective cohort study. Diabetologia. 2017 Oct;60(10):2092-102.

19 Zahn D, Herpertz S, Albus C, Hermanns N, Hiemke C, Hiller W, et al. hs-CRP predicts improvement in depression in patients with type 1 diabetes and major depression undergoing depression treatment: results from the Diabetes and Depression (DAD) study. Diabetes Care. 2016 Oct;39(10):e171-3.

20 Schmitt A, Reimer A, Kulzer B, Haak T, Ehrmann D, Hermanns N. How to assess diabetes distress: comparison of the Problem Areas in Diabetes Scale (PAID) and the Diabetes Distress Scale (DDS). Diabet Med. 2016 Jun; 33(6):835-43.

21 Snoek FJ, Bremmer MA, Hermanns N. Constructs of depression and distress in diabetes: time for an appraisal. Lancet Diabetes Endocrinol. 2015 Jun;3(6):450-60.

22 Aikens JE. Prospective associations between emotional distress and poor outcomes in type 2 diabetes. Diabetes Care. 2012 Dec;35(12): 2472-8.

23 Schmitt A, Reimer A, Kulzer B, Haak T, Gahr A, Hermanns N. Negative association between depression and diabetes control only when accompanied by diabetes-specific distress. J Behav Med. 2015 Jun;38(3):556-64.

24 Hermanns N, Schmitt A, Gahr A, Herder C, Nowotny B, Roden M, et al. The effect of a diabetes-specific cognitive behavioral treatment program (DIAMOS) for patients with diabetes and subclinical depression: results of a randomized controlled trial. Diabetes Care. 2015 Apr;38(4):551-60.
25 Young-Hyman D, De Groot M, Hill-Briggs F, Gonzalez JS, Hood K, Peyrot M. Psychosocial care for people with diabetes: a position statement of the American Diabetes Association. Diabetes Care. 2016 Dec;39(12):2126-40.

26 Diabetes Canada Clinical Practice Guidelines Expert Committee; Robinson DJ, Coons M, Haensel H, Vallis M, Yale JF. Diabetes and mental health. Can J Diabetes. 2018 Apr;42 Suppl 1:S130-41.

27 Petrak F, Baumeister H, Skinner TC, Brown A, Holt RIG. Depression and diabetes: treatment and health-care delivery. Lancet Diabetes Endocrinol. 2015 Jun;3(6):472-85.

28 Reimer A, Schmitt A, Ehrmann D, Kulzer B, Hermanns N. Reduction of diabetes-related distress predicts improved depressive symptoms: a secondary analysis of the DIAMOS study. PLoS One. 2017 Jul;12(7):e0181218.

29 Guidi J, Brakemeier EL, Bockting CLH, Cosci F, Cuijpers P, Jarrett RB, et al. Methodological recommendations for trials of psychological interventions. Psychother Psychosom. 2018 Sep;87:276-84.

30 Williams JB, Kobak KA, Bech P, Engelhardt N, Evans K, Lipsitz J, et al. The GRID-HAMD: standardization of the Hamilton Depression Rating Scale. Int Clin Psychopharmacol. 2008 May;23(3):120-9.

31 Schmitt A, Kulzer B, Hermanns N. German version of the GRID Hamilton Rating Scale for Depression (GRID-HAMD). Sep 2015.

32 Carrozzino D, Patierno C, Fava GA, Guidi J. The Hamilton rating scales for depression: a critical review of clinimetric properties of different versions. Psychother Psychosom. 2020; 89(3):133-50.

33 DGPPN, BÄK, KBV, AWMF. S3-Leitlinie/ Nationale Versorgungsleitlinie Unipolare Depression: Langfassung. 2nd ed. Version 5. Berlin: Springer; 2015. p. 177.

34 Hautzinger M, Bailer M, Hofmeister D, Keller F. ADS: Allgemeine Depressionsskala (2. überarbeitete, neu normierte Auflage) [General depression scale]. 2nd ed., revised edition. Göttingen: Hogrefe; 2012.

35 Polonsky WH, Anderson BJ, Lohrer PA, Welch G, Jacobson AM, Aponte JE, et al. Assessment of diabetes-related distress. Diabetes Care. 1995 Jun;18(6):754-60.

36 Laux L, Glanzmann P, Schaffner P, Spielberger CD. STAI: das State-trait angstinventar. 1. Auflage 1981 [STAI: the State-Trait Anxiety Inventory. 1st ed. Göttingen: Beltz Test; 1981.

37 Topp CW, Østergaard SD, Søndergaard S, Bech P. The WHO-5 well-being index: a systematic review of the literature. Psychother Psychosom. 2015 Apr;84(3):167-76.

38 Schmitt A, Reimer A, Kulzer B, Haak T, Gahr A, Hermanns N. Assessment of diabetes acceptance can help identify patients with ineffective diabetes self-care and poor diabetes control. Diabet Med. 2014 Nov;31(11):144651.
Stepped Care Approach for Depression and Distress in Diabetes
Psychother Psychosom 2022;91:107-122 DOI: $10.1159 / 000520319$ 
39 Johnson JA, Coons SJ. Comparison of the EQ$5 \mathrm{D}$ and SF-12 in an adult US sample. Qual Life Res. 1998 Feb;7(2):155-66.

40 Toobert DJ, Hampson SE, Glasgow RE. The summary of diabetes self-care activities measure: results from 7 studies and a revised scale. Diabetes Care. 2000 Jul;23(7):943-50.
41 Schmitt A, Reimer A, Hermanns N, Huber J, Ehrmann D, Schall S, et al. Assessing diabetes self-management with the diabetes self-management questionnaire (DSMQ) can help analyse behavioural problems related to reduced glycaemic control. PLoS One. 2016 Mar;11(3):e0150774.

42 Harb BM, Kubiak T, Roth R. Diabetes treatment satisfaction questionnaire (DTSQ). In: Bengel J, Wirtz M, Zwingmann Z, editors. Diagnostische verfahren in der rehabilitation. Göttingen: Hogrefe; 2008. p. 266-9.
43 Carrozzino D, Patierno C, Guidi J, Berrocal Montiel C, Cao J, Charlson ME, et al. Clinimetric criteria for patient-reported outcome measures. Psychother Psychosom. 2021; 90(4):222-32.

44 Baumeister H, Hutter N, Bengel J. Psychological and pharmacological interventions for depression in patients with diabetes mellitus: an abridged Cochrane review. Diabet Med. 2014 Jul;31(7):773-86 